\title{
High Frequency of Antimicrobial Drug Resistance of Diarrheagenic Escherichia coli in Infants in Peru
}

\author{
Theresa J. Ochoa,* Joaquím Ruiz, Margarita Molina, Luis J. Del Valle, Martha Vargas, Ana I. Gil, Lucie Ecker, \\ Francesca Barletta, Eric Hall, Thomas G. Cleary, and Claudio F. Lanata \\ Instituto de Medicina Tropical, Universidad Peruana Cayetano Heredia, Lima, Peru; Center for Infectious Diseases, University of Texas School of \\ Public Health, Houston, Texas; Centre de Recerca en Salut Internacional de Barcelona, Hospital Clinic/Institut d'Investigacions Biomèdiques \\ August Pi i Sunyer, Barcelona, Spain; Instituto de Investigación Nutricional, Lima, Peru; Departament d'Enginyeria Agroalimentària $i$ \\ Biotecnologia, Escola Superior d'Agricultura de Barcelona, Universitat Politécnica de Catalunya, Castelldefels, Spain; U.S. Naval \\ Medical Research Center, Lima, Peru; Escuela de Medicina, Universidad Peruana de Ciencias Aplicadas, Lima, Peru
}

Abstract. In a prospective passive diarrhea surveillance cohort study of 1,034 infants of low socioeconomic communities in Lima, Peru, we determined the prevalence and antimicrobial drug susceptibility of the diarrheagenic Escherichia coli. The prevalence of diarrheagenic E. coli was 29\% (161 of 557) in children with gastroenteritis and $30 \%$ (58 of 195) in the control group without diarrhea. The most common E. coli pathogens in diarrhea were enteroaggregative E. coli (EAEC) (14\%), enteropathogenic E. coli (EPEC) (7\%), diffusely adherent E. coli (DAEC) (4\%), and enterotoxigenic E. coli (ETEC) (4\%). Diarrheagenic E. coli as a group exhibited high levels of antimicrobial drug resistance in diarrheal cases to ampicillin $(85 \%)$, cotrimoxazole $(79 \%)$, tetracycline $(65 \%)$, and nalidixic acid $(28 \%)$. Among individual E. coli groups in patients with diarrhea, DAEC and EAEC exhibited significant higher frequencies of resistance to ampicillin, cotrimoxazole, tetracycline and nalidixic acid than EPEC and ETEC. Antimicrobial drug resistance to ampicillin and cotrimoxazole were more frequent in E. coli isolated from diarrheal samples than controls, which reflected greater antibiotic exposure in patients with gastroenteritis.

\section{INTRODUCTION}

Diarrheal illness remains one of the leading causes of morbidity and mortality worldwide, despite ongoing progress in our basic understanding of its epidemiology, pathogenesis, and treatment. In developing areas, infectious diarrhea is a major cause of childhood mortality; there are an estimated 2 million deaths/year among children globally. ${ }^{1}$ Beyond this, however, are its under-recognized long-term effects, which include permanent shortfalls in physical and cognitive development attributable to early repeated childhood diarrhea episodes and to enteric parasitic infections. ${ }^{2-6}$ The longitudinal prevalence of diarrhea seems to be a stronger predictor of the long-term health effects of diarrhea than the incidence rates. ${ }^{7}$ Therefore, the duration of the diarrheal episodes in small children may be critical.

Diarrheagenic Escherichia coli are major causes of gastroenteritis in children in the developing world and are associated with high resistance levels to antibiotics. ${ }^{8-11}$ Escherichia coli associated with diarrhea have been classified into six groups based on clinical, epidemiologic, and molecular criteria: ${ }^{12}$ enteropathogenic E. coli (EPEC); enterotoxigenic E. coli (ETEC); Shiga toxin-producing E. coli (STEC), also known as enterohemorrhagic E. coli (EHEC) or verotoxin-producing E. coli (VTEC); 4 enteroinvasive E. coli (EIEC); enteroaggregative E. coli (EAEC or EAggEC); and diffusely adherent E. coli (DAEC). The course of illness with many of these pathogens might be ameliorated by specific antimicrobial drug therapy. However, specific etiologic diagnosis, as well as the knowledge of the local antibiotic susceptibility patterns, is necessary if treatment interventions are to be considered. Our hypothesis was that because of frequent inappropriate empiric antibiotic use in Lima, there should be a high prevalence of

\footnotetext{
* Address correspondence to Theresa J. Ochoa, Instituto de Medicina Tropical Alexander von Humboldt, Universidad Peruana Cayetano Heredia, Avenida Honorio Delgado 430, San Martin de Porras, Lima 31, Peru. E-mail: theresa.j.ochoa@uth.tmc.edu
}

antimicrobial drug resistance in strains of diarrheagenic E. coli isolated from children whether the strains were isolated from infants with diarrhea or controls without diarrhea.

The objective of this study was to determine the prevalence, antimicrobial drug susceptibility patterns, and association of these patterns with specific classes of diarrheagenic E. coli. Such information might be useful both in discouraging inappropriate use and guiding physicians to more appropriate choices when therapy is necessary. We focused on infants in the first year of life to determine whether resistant organisms were acquired very early in life.

\section{PATIENTS AND METHODS}

This study was a prospective passive diarrhea surveillance cohort study of 1,034 infants in Peru followed-up from 2 to 12 months of age in low socioeconomic communities in the southern districts of Lima, Peru. A complete description of the patients and methods of the cohort diarrhea study is presented elsewhere (Ochoa TJ and others, unpublished data). Children were enrolled in the study after informed consent was obtained from the parents of the infants. The study was reviewed and approved by the Ethical Review Board of the Instituto de Investigación Nutricional and Universidad Peruana Cayetano Heredia, Lima, Perú. Diarrheal episodes that required medical attention at our study clinic were evaluated. Treatment advice was provided to parents, including use of oral rehydration solution and antibiotics if needed, as well as advice regarding dietary management. Additionally, control stool samples were obtained monthly from 20-30 randomly selected healthy study children; these children had no diarrhea for at least seven days before and seven days after the stool sample collection. Children were followed-up at home one week later to confirm the absence of diarrhea after the control stool samples was taken.

Stool samples were analyzed for the presence of common enteric viruses, bacteria, and parasites by using conventional methods. Five lactose-fermenting colonies were selected from 
each MacConkey plate for analysis for the presence of the diarrheagenic E. coli. Detection was conducted by using a realtime multiplex polymerase chain reaction system ${ }^{13}$ with primers described in Table 1 and a PTC-200 thermal cycler with chromo 4 optical detector (MJ Research/Bio-Rad, Hercules, CA). Escherichia coli strains positive for any diarrheagenic E. coli gene were analyzed for their antimicrobial drug susceptibility by disk diffusion according to the Clinical Laboratory Standards Institute guidelines. ${ }^{14}$ The antibiotics analyzed were ampicillin (10- $\mu \mathrm{g}$ disk), amoxicillin-clavulanic acid (30 $\mu \mathrm{g}$-disk), azythromycin (15- $\mu \mathrm{g}$ disk), cefotaxime (30- $\mu \mathrm{g}$ disk), ceftazidime (30- $\mu \mathrm{g}$ disk), chloramphenicol (30- $\mu \mathrm{g}$ disk), ciprofloxacin (5- $\mu \mathrm{g}$ disk), cotrimoxazole (23.75/1.25- $\mu \mathrm{g}$ disk), gentamicin $(10-\mu \mathrm{g}$ disk), nalidixic acid $(30-\mu \mathrm{g}$ disk), nitrofurantoin (300- $\mu \mathrm{g}$ disk), and tetracycline $(30-\mu \mathrm{g}$ disk).

The overall prevalence of each type of diarrheagenic E. coli in gastroenteritis and control stool samples, prevalence by age groups, and antibiotic resistance rates were compared. Statistical differences were evaluated by chi-square or Fisher's exact tests.

\section{RESULTS}

The diarrhea surveillance study for antimicrobial drug susceptibility was conducted from September 2006 through May 2007. During this 8-month period, we studied 557 stool samples from children with diarrhea and 195 control samples from children without diarrhea. Some children had more than one episode of diarrhea requiring medical attention during the study period and some had none. The mean \pm SD age of the children studied was $5.2 \pm 1.8$ months; the oldest child in this group was 10 months of age. The prevalence of the diarrheagenic E. coli was $29 \%$ (161 of 557) in the diarrhea group and $30 \%$ (58 of 195) in the control group. The prevalence of each diarrheagenic $E$. coli group in diarrhea and control samples was EAEC 14\% (78 of 557) versus 18\% (35 of 195$)$, EPEC 7\% (37 of 557) versus 7\% (13 of 195), DAEC $4 \%$ (21 of 557) versus 3\% (6 of 195), ETEC $4 \%$ (20 of 557 ) versus $2 \%$ (3 of 195), and STEC 1\% (5 of 557) versus $0.5 \%$ (1 of 195). No EIEC strains were isolated. The prevalence of other enteric pathogens in diarrhea and control samples was
Campylobacter spp. 14\% (78 of 557) versus 8\% (16 of 195), Salmonella spp. $0 \%$ versus $0.5 \%$ (1 of 195 ). Rotavirus was found in $7 \%$ (37 of 548) of diarrhea samples; control samples were not tested for rotavirus. There were no Shigella spp. or Vibrio cholerae isolated.

The inappropriate use of antibiotics during illnesses was common in this population. Children from the diarrhea group received more courses of antibiotics (for any illness) in the previous three months prior to the stool sample collection than children in the control group $(1.0 \pm 1.3$ courses of antibiotics/ child versus $0.6 \pm 0.8$, respectively, $P=0.023)$. The most common antibiotics used were amoxicillin $(31 \%$ versus $42 \% ; P=$ 0.050 ), macrolides ( $32 \%$ versus $19 \% ; P=0.032$ ), and cotrimoxazole $(15 \%$ versus $23 \% ; P=0.076)$ in the diarrhea and control groups, respectively ( 288 courses of antibiotics in the diarrhea group and 78 courses in the control group). Empiric antibiotic (selected by the parents without medical advice or prescribed by a physician) was used in $46 \%$ of diarrhea episodes in which a diarrheagenic E. coli was isolated. The most commonly used antibiotics for diarrhea episodes were macrolides 26\% (33 of 129) (erythromycin $20 \%$, azythromycin $6 \%$ ), furazolidone $8 \%$ (10 of 129), amoxicillin 5\% (7 of 129), and cotrimoxazole $2 \%$ (3 of 129).

Eighty percent of the diarrheagenic E. coli from diarrhea cases (129 of 161) and 98\% of the control samples (57 of 58) were available for the antibiotic susceptibility studies. The diarrheagenic $E$. coli as a group were frequently resistant to ampicillin, cotrimoxazole, tetracycline, nalidixic acid, and chloramphenicol (Table 2). All strains were fully susceptible to cefotaxime and ceftazidime. There were no strains with high-level resistance to amoxicillin/clavulanic. However, $12 \%$ had intermediate resistance among diarrheal samples and $9 \%$ among controls. Similarly, $13 \%$ of strains showed intermediate resistance to nitrofurantoin among diarrheal samples and $2 \%$ in controls. Although azythromycin is commonly used as therapy for some enteric pathogens, there are no approved resistance criteria for disk-diffusion analysis of $E$. coli. The distribution of the growth inhibitory zones by disk diffusion in 167 diarrheagenic E. coli from diarrhea and control samples were $10 \%$ (17 strains) had azythromycin inhibitory diameter

TABLE 1

Primers for multiplex real-time polymerse chain reaction for diarrheagenic Escherichia coli genes*

\begin{tabular}{|c|c|c|c|c|}
\hline Group & Gene & Primer sequence $\left(5^{\prime} \rightarrow 3^{\prime}\right) \dagger$ & Size (basepairs) & $\operatorname{Tm} \ddagger\left(C^{\circ}\right)$ \\
\hline \multirow[t]{2}{*}{ EAEC } & $\operatorname{aggR}$ & F CGAAAAAGAGATTATAAAAATTAAC & 100 & 77 \\
\hline & & R GCTTCCTTCTTTTGTGTAT & & \\
\hline \multirow[t]{5}{*}{ ETEC } & stIa & F TTTCСССТCTTTTAGTCAGTCAA & 159 & 81 \\
\hline & $s t I b$ & F TGCTAAACCAGTAGAGTCTTCAAAA & 138 & \\
\hline & st & R GCAGGATTACAACACAATTCACAGCAG & & \\
\hline & $l t$ & F $\quad$ TCTCTATGTGCATACGGAGC & 322 & 86 \\
\hline & & R CCATACTGATTGCCGCAAT & & \\
\hline \multirow[t]{2}{*}{ EPEC } & eaeA & F ATGCTTAGTGCTGGTTTAGG & 248 & 83 \\
\hline & & R GCCTTCATCATTTCGCTTTC & & \\
\hline \multirow[t]{4}{*}{ STEC $\S$} & stxl & F CTGGATTTAATGTCGCATAGTG & 150 & 87 \\
\hline & & R AGAACGCCCACTGAGATCATC & & \\
\hline & stx 2 & F GGCACTGTCTGAAACTGCTCC & 255 & 89 \\
\hline & & R TCGCCAGTTATCTGACATTCTG & & \\
\hline \multirow[t]{2}{*}{ EIEC } & ipaH & F GTTCCTTGACCGCCTTTCCGATACCGTC & 619 & 91 \\
\hline & & R GCCGGTCAGCCACCCTCTGAGAGTAC & & \\
\hline \multirow[t]{2}{*}{ DAEC } & daaD & F TGAACGGGAGTATAAGGAAGATG & 444 & 93 \\
\hline & & R GTCCGCCATCACATCAAAA & & \\
\hline
\end{tabular}

*EAEC = enteroaggregative E. coli $; \mathrm{F}=$ forward $; \mathrm{R}=$ reverse $; \mathrm{ETEC}=$ enterotoxigenic $E$. coli $; \mathrm{EPEC}=$ enteropathogenic $E$. coli $; \mathrm{STEC}=\mathrm{Shiga}$ toxin-producing $E$. coli $;$ EIEC $=$ enteroinvasive

E. coli; DAEC $=$ diffusely adherent $E$. coli.

$\dagger$ Primers described by Guion and others. ${ }^{13}$

$\$ \mathrm{Tm}=$ melting temperature for each amplicon.

\$STEC strains could be eaeA positive and should always have $s t x 1$ or $s t x 2$ or $s t x 1$ and $s t x 2$. 
TABLE 2

Antibiotic resistance rates of diarrheagenic Escherichia coli from diarrhea $(\mathrm{n}=129)$ and control $(\mathrm{n}=55)$ samples*

\begin{tabular}{|c|c|c|c|c|c|c|c|c|}
\hline \multirow[b]{2}{*}{ Drug } & \multicolumn{2}{|c|}{ EAEC, no. $(\%)$} & \multicolumn{2}{|c|}{ EPEC, no. (\%) } & \multicolumn{2}{|c|}{ ETEC, no. (\%) } & \multicolumn{2}{|c|}{ DAEC, no. (\%) } \\
\hline & Diarrhea $(\mathrm{n}=67)$ & Control $(\mathrm{n}=34)$ & Diarrhea $(\mathrm{n}=32)$ & Control $(\mathrm{n}=12)$ & Diarrhea $(\mathrm{n}=18)$ & Control $(\mathrm{n}=3)$ & Diarrhea $(\mathrm{n}=12)$ & Control $(n=6)$ \\
\hline Ampicillin & $61(91)$ & $26(76)$ & $23(72)$ & $5(42)$ & $14(78)$ & $2(67)$ & $12(100)$ & $6(100)$ \\
\hline Cotrimoxazole & $57(85)$ & $22(65)$ & $23(72)$ & $3(25)$ & $11(61)$ & $3(100)$ & $11(92)$ & $6(100)$ \\
\hline Tetracycline & $49(73)$ & $22(65)$ & $16(50)$ & $2(17)$ & $8(44)$ & $2(67)$ & $11(92)$ & $6(100)$ \\
\hline Nalidixic Acid & $22(33)$ & $5(15)$ & 7 (22) & $1(8)$ & $1(6)$ & $0(0)$ & $6(50)$ & $2(33)$ \\
\hline Chloramphenicol & $26(39)$ & $13(38)$ & $2(6)$ & $0(0)$ & $1(6)$ & $0(0)$ & $5(42)$ & $3(50)$ \\
\hline Gentamicin & $4(6)$ & $1(3)$ & $0(0)$ & $0(0)$ & $0(0)$ & $0(0)$ & $1(8)$ & $0(0)$ \\
\hline Nitrofurantoin & $0(0)$ & $0(0)$ & $0(0)$ & $1(8)$ & $3(17)$ & $0(0)$ & $0(0)$ & $0(0)$ \\
\hline Ciprofloxacin & $1(1)$ & $0(0)$ & $0(0)$ & $0(0)$ & $0(0)$ & $0(0)$ & $1(8)$ & $1(17)$ \\
\hline Multi-resistance $\dagger$ & $47(70)$ & $19(57)$ & $15(47)$ & $2(17)$ & $7(39)$ & $2(67)$ & $12(100)$ & $6(100)$ \\
\hline
\end{tabular}

*Data on Shiga toxin-producing E. coli are not presented because of a small number of samples. EAEC = enteroaggregative E. coli; EPEC = enteropathogenic E. coli; ETEC = enterotoxigenic E. coli; DAEC = diffusely adherent E. coli.

+ coli $;$ DAEC $=$ diffusely adherent $E . c o l i$.
$\dagger$ Resistance to 3 or more antibiotics.

zones less than $10 \mathrm{~mm}, 21 \%$ (35 strains) had diameters $11-15$ $\mathrm{mm}, 33 \%$ (55 strains) had diameters 16-20 mm, and 36\% (60 strains) had diameters more than $21 \mathrm{~mm}$.

There was a higher frequency of resistance to all antibiotics in diarrheal samples than in controls; this difference was significant for ampicillin ( $85 \%$ versus $70 \% ; P=0.039)$ and cotrimoxazole ( $79 \%$ versus $61 \% ; P=0.024$ ) (Figure 1$)$. Multidrug resistance, defined as resistance to three or more antibiotics, was common in diarrheal $(63 \%, 81$ of 129$)$ and control samples $(53 \%, 29$ of 57$)$. There were 14 different antibiotic resistance patterns among the diarrheagenic E. coli (diarrhea and control strains grouped together) (Table 3). The most common resistance patterns were ampicillin-cotrimoxazole-tetracycline, present in $24 \%$ of all strains (41 of 168), and ampicillincotrimoxazole, present in $19 \%$ of strains (25 of 168).

Among the individual E. coli groups, there was a striking difference in susceptibility between them. DAEC consistently exhibited more resistance to ampicillin, cotrimoxazole, tetracycline, and nalidix acid than EAEC, which was more resistant than EPEC, which was more resistant than ETEC. This trend was significant for all four antibiotics (Figure 2). Multiple resistance also differed strikingly with DAEC and EAEC much more resistant (Table 3) than the other categories of E. coli. Resistant to three or more agents was DAEC (100\%), EAEC (70\%), ETEC (47\%), and EPEC (44\%) $(P=0.0001)$.

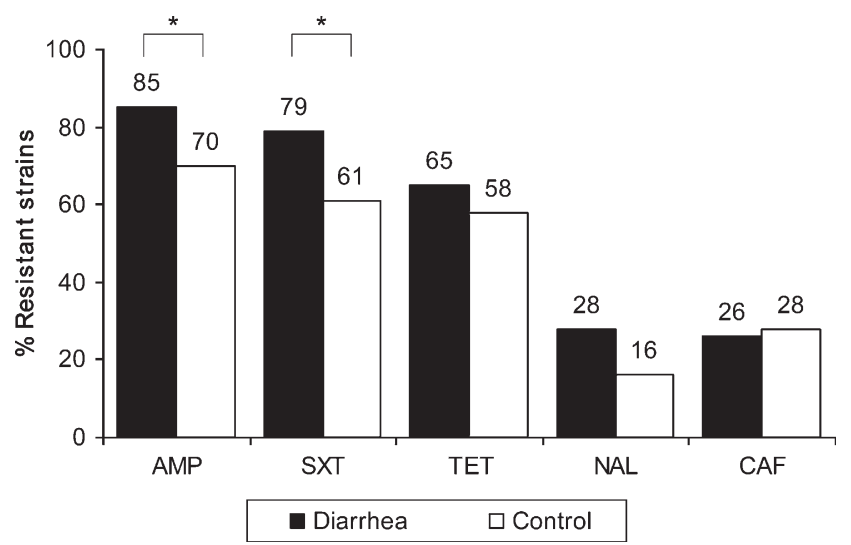

Figure 1. Antibiotic resistance of diarrheagenic Escherichia coli as a group in infants with diarrhea $(\mathrm{n}=129)$ and without diarrhea $(\mathrm{n}=57)$. Shown is the percentage of strains with high-level resistance by the disk-diffusion method. $\mathrm{AMP}=$ ampicillin; $\mathrm{SXT}=$ cotrimoxazole; TET $=$ tetracycline $; \mathrm{NAL}=$ nalidixic acid $; \mathrm{CAF}=$ chloramphenicol . ${ }^{*} P<0.05$ for the comparison between diarrhea and control.

\section{DISCUSSION}

For many episodes of infectious diarrhea, only fluid and electrolyte management is appropriate. However, antimicrobial drug therapy is indicated for children and adults with acute infectious gastroenteritis in a variety of circumstances. ${ }^{15}$ These indications may include dysentery, severe or prolonged disease, eradication of fecal shedding and transmission, and prevention of sequellae and death. ${ }^{15}$ However, antimicrobial agents do not benefit most children with acute diarrhea because viral enteropathogens are so common. Thus, in children with acute gastroenteritis, use of specific antimicrobial drugs should be limited to well-defined bacterial and protozoal agents. Among the diarrheagenic E. coli, there is an established benefit for the use of antibiotics for ETEC, EIEC and EAEC infections; these conclusions are based primarily on data in traveler's diarrhea. ${ }^{16}$ However, the role of antibiotic therapy in children with acute diarrhea caused by a diarrheagenic $E$. coli is not fully defined. ${ }^{17}$ This finding is caused primarily by the lack of rapid diagnostic tests available at the outpatient or hospital settings. ${ }^{18}$ The time frame in which treatment choices must be made is short. Prior to the development of the mulitplex real time polymerase chain reaction, there was no rapid, sensitive, and inexpensive diagnostic technique for determining presence of a diarrheagenic E. coli in the community setting. Now that such rapid assays are available in research laboratories, local epidemiologic studies on etiology and antimicrobial drug susceptibility are relevant.

Diarrheagenic E. coli were the most commonly isolated pathogens in infants in Peru with diarrhea. This finding is similar to those of studies from other developing countries, where the diarrheagenic E. coli as a group are responsible for 30-40\% of acute diarrhea episodes in children. ${ }^{18}$ However, in this study, the diarrheagenic $E$. coli were found at similar frequencies in children with and without diarrhea, demonstrating that infants in this setting where breastfeeding is universal are frequently exposed to these bacteria. This study clearly demonstrates that multiply resistant organisms are acquired very early in life.

These organisms are exposed to antibiotic pressure when both enteric and non-enteric infections occur. In this study, antimicrobial drug resistance in $E$. coli was associated with a high frequency of antibiotic use for diarrhea (46\%) and with a higher frequency of previous antibiotic exposure for nonenteric infections. Recent antibiotic use, particularly one month before exposure, is a risk factor for developing infection or colonization with resistant bacterial pathogens. ${ }^{19,20}$ Excessive and inappropriate use of antimicrobial drugs for 
TABLE 3

Antibiotic resistance patterns for the diarrheagenic Escherichia coli $(\mathrm{n}=168)$ from diarrhea and control samples*

\begin{tabular}{|c|c|c|c|c|c|}
\hline Pattern no. & Antibiotic resistance patterns & $\operatorname{EAEC~}(\mathrm{n}=94)$ & $\operatorname{EPEC}(\mathrm{n}=39)$ & $\operatorname{DAEC}(\mathrm{n}=18)$ & $\operatorname{ETEC}(\mathrm{n}=17)$ \\
\hline I & AMP-SXT-TET-NAL-GTM-CIP & 1 & 0 & 0 & 0 \\
\hline II & AMP-SXT-TET-NAL-CAF & 19 & 1 & 3 & 0 \\
\hline II & AMP-SXT-TET-CAF-GTM & 3 & 0 & 2 & 0 \\
\hline IV & AMP-SXT-TET-NAL-CIP & 0 & 0 & 2 & 0 \\
\hline V & AMP-SXT-TET-CAF & 13 & 1 & 3 & 0 \\
\hline VI & AMP-SXT-TET-NAL & 4 & 3 & 0 & 0 \\
\hline VII & AMP-SXT-TET-NIT & 0 & 0 & 0 & 3 \\
\hline VIII & AMP-SXT-CAF-GTM & 2 & 0 & 0 & 0 \\
\hline IX & AMP-TET-NAL-CAF & 0 & 0 & 1 & 0 \\
\hline $\mathrm{X}$ & SXT-TET-NAL-CIP & 1 & 0 & 0 & 0 \\
\hline XI & AMP-SXT-TET & 18 & 12 & 6 & 5 \\
\hline XII & AMP-SXT-NAL & 3 & 0 & 0 & 0 \\
\hline XIII & AMP-SXT-CAF & 1 & 0 & 1 & 0 \\
\hline \multirow[t]{4}{*}{ XIV } & AMP-TET-NAL & 1 & 0 & 0 & 0 \\
\hline & Resistance to two antibiotics $\dagger$ & 17 & 10 & 0 & 5 \\
\hline & Resistance to only one antibiotic & 6 & 6 & 0 & 3 \\
\hline & Pan-susceptible & 5 & 6 & 0 & 1 \\
\hline
\end{tabular}

$* \mathrm{EAEC}=$ enteroaggregative $E$. coli $; \mathrm{EPEC}=$ enteropathogenic $E$. coli $; \mathrm{DAEC}=$ diffusely adherent $E$. coli $; \mathrm{ETEC}=$ enterotoxigenic $E$. coli $; \mathrm{AMP}=$ ampicillin $; \mathrm{SXT}=$ cotrimoxazole $; \mathrm{TET}=$ tet racycline; $\mathrm{NAL}=$ nalidixic acid $; \mathrm{GTM}=$ gentamicin $; \mathrm{CIP}=$ ciprofloxacin $; \mathrm{CAF}=$ chloramphenicol, $\mathrm{NIT}=$ nitrofurantoin.

$\dagger$ Resistance patterns: AMP-SXT (25 strains), AMP-TET ( 3 strains), SXT-TET ( 2 strains), SXT-NAL (1 strain), and TET-NAL (1 strain).

proven or presumed infections in many organ systems, including the gastrointestinal tract, is a common problem. This use occurs in part because of inappropriate prescribing practices and in part because of misguided beliefs and expectations of parents who often lack an awareness of the dangers of antimicrobial drug use. ${ }^{19}$ In Peru, as in many other developing countries, antimicrobial drugs are sold over the counter without a prescription. Thus, unrestricted accesses to antimicrobial drugs coupled with lack of understanding regarding their use are major factors driving multiresistance. ${ }^{21-23}$

The problem of antimicrobial drug resistance is not unique to Peru. It is particularly critical in many developing countries where frequent illnesses coupled with ready access to unregulated antibiotics diminishes the value of these agents for those patients who actually need them. We found a high frequency of antimicrobial resistance of diarrheagenic E. coli to commonly used antibiotics such as ampicillin (85\%) and cotrimoxazole (79\%). This finding is similar to what has been recently described in children from Vietnam $(86 \%$ and $88 \%$,

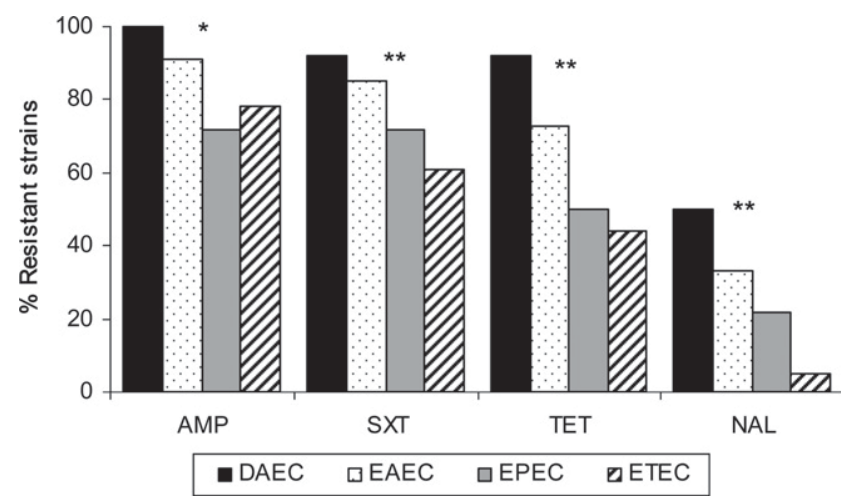

FIGURE 2. Antibiotic resistance among the different diarrheagenic Escherichia coli groups in infants with diarrhea. DAEC = diffusely adherent E. coli $(\mathrm{n}=12) ;$ EAEC = enteroaggregative E. coli $(\mathrm{n}=67)$, EPEC $=$ enteropathogenic E. coli $(\mathrm{n}=32) ; \mathrm{ETEC}=$ enterotoxigenic E. coli $(\mathrm{n}=18)$. AMP = ampicillin; $\mathrm{SXT}=$ cotrimoxazole; $\mathrm{TET}=$ tet racycline; NAL $=$ nalidixic acid. $* P<0.05 ; * * P<0.01$ (by chi-square test for trend) for comparison of the resistance percentage among the different E. coli. respectively), ${ }^{10}$ Tanzania ( $85 \%$ and $\left.87 \%\right),{ }^{8}$ México $(73 \%$ and $65 \%),{ }^{11}$ Argentina (75\% and 64\%) $)^{24}$ and Mozambique $(72 \%$ and $58 \%) .{ }^{25}$ Of interest, ampicillin or cotrimoxazole are not appropriate empiric therapy for common enteric bacteria such as Shigella spp. and Salmonella spp. because of the high frequency of resistance in many areas of the world..$^{15}$ The growing problem of multidrug-resistant enteric pathogens is especially common in Africa and Asia. ${ }^{26-28}$

Resistance patterns were strikingly different among individual E. coli groups. DAEC and EAEC had higher resistance levels than EPEC and ETEC. This finding is likely to result from several factors. It is possible that DAEC and EAEC are more resistant because they are exposed to antimicrobial drugs more often, which may be because they cause persistent diarrhea and/or are often carried asymptomatically. Thus, the long time within human hosts increases the chance that they will be exposed to antimicrobial drugs and/or acquire resistant genes from the resident flora. These data could also reflect an association of resistance genes with plasmid-associated virulence genes, such as adherence factors present in DAEC (i.e., Dr adhesins) ${ }^{29}$ and EAEC (i.e., aggregative adherence fimbria). ${ }^{30}$ It is known that resistance to multiple antibiotics can be caused by a variety of mobile genetic elements such as plasmids, transposons, and gene cassettes in integrons, ${ }^{31}$ or to alteration in the $E$. coli multiple antibiotic resistance operon (mar). ${ }^{32}$ The Mar phenotype includes resistance to structurally unrelated antibiotics such as tetracycline, chloramphenicol, $\beta$-lactams, fluoroquinolones, puromycin, nalidixic acid, rifampicin, and others. ${ }^{33}$ Integron-associated antibiotic resistance has been described for EIEC, EAEC, and cell-detaching $E$. coli, ${ }^{34,35}$ and a conjugative multi-resistance plasmid has been reported for EPEC. ${ }^{36}$

In summary, diarrheagenic E. coli are the most common isolated pathogens in infants in Peru with diarrhea and in asymptomatic controls, which reflects the high and early exposure to pathogens in this setting. Diarrheagenic E. coli were associated with a high frequency of antibiotic resistance, with different resistance patterns among the individual E. coli groups. Overall, resistance to ampicillin and cotrimoxazole were so common that both drugs should not be use empirical for the treatment of invasive or persistent bacterial gastroenteritis 
in children from Lima. Antimicrobial drug resistance was more frequent in E. coli isolated from diarrheal samples than from controls and associated with more antibiotic exposure in patients with gastroenteritis. The impact of unsupervised antibiotic use is clear. Inappropriate antibiotics are expensive, potentially toxic, used with dubious indication that is unlikely to result in benefit to the recipient, and select organisms that are multiply resistant so that the usefulness of the agents is limited when legitimate medical indications exist. It is imperative to implement strategies to prevent and control the emergence and spread of resistant organisms by improving diagnosis and reducing the selective pressure caused by overuse and misuse of antibiotics in children.

Received September 19, 2008. Accepted for publication May 12, 2009.

Financial support: This study was partially supported by the Agencia Española de Cooperación Internacional (grant A/4892/06), the Agència Catalana de Cooperació al Desenvolupament, Generalitat de Catalunya (grant U2006), and the Centre de Cooperació per al Desenvolupament, Universitat Politècnica de Catalunya (grant to Luis J. Del Valle LJdV). Theresa J. Ochoa is supported by grant PHS-FIC1K01TW007405 from the National Institutes of Health, Joaquím Ruiz is supported by grant FIS CP05/0130 from the Fondo de Investigaciones Sanitarias, Spain, Thomas G. Cleary is supported by grant PHS-NICHD R01-HD051716 from the National Institutes of Health.

Disclaimer: The views expressed in this article are those of the author and do not necessarily reflect the official policy or position of the Department of the Navy, Department of Defense, the U.S. Government, or the National Institutes of Health and other funding institutions.

Authors' addresses: Theresa J. Ochoa and Francesca Barletta, Instituto de Medicina Tropical Alexander von Humboldt, Universidad Peruana Cayetano Heredia, Avenida Honorio Delgado 430, San Martin de Porras, Lima 31, Peru, E-mails: theresa.j.ochoa@uth.tmc.edu and francescabarletta@yahoo.es. Joaquím Ruiz, Centre de Recerca en Salut Internacional de Barcelona, C/Rosselló 132, 4.08036, Barcelona, Spain, E-mail: joruiz@clinic.ub.es. Margarita Molina, Ana I. Gil, Lucie Ecker, and Claudio F. Lanata, Instituto de Investigación Nutricional, Avenida La Molina 1885, La Molina, Lima 12, Peru, E-mails: micro@iin.sld.pe, agil@iin.sld.pe, lecker@iin.sld.pe, and clanata@iin.sld.pe. Luis J. del Valle, Departament d'Enginyeria Agroalimentària i Biotecnologia, Escola Superior d'Agricultura de Barcelona, Universitat Politècnica de Catalunya, Avenida Canal Olímpic 15, 08860 Castelldefels, Barcelona, Spain,E-mail:luis.javier.del.valle@upc.edu.Martha Vargas, Hospital Clinic de Barcelona Villarroel 179, 08036 Barcelona, Spain, E-mail: mvargas@clinic.ub.es. Eric Hall, Director for Administration, Public Affairs Officer, Naval Medical Research Center, 503 Robert Grant Avenue, Silver Spring, MD, E-mail: eric.hall@med.navy.mil. Thomas G. Cleary, Center for Infectious Diseases, University of Texas School of Public Health, PO Box 20186, Houston, TX 77225, E-mail: thomas.g.cleary@uth.tmc.edu.

\section{REFERENCES}

1. Bryce J, Boschi-Pinto C, Shibuya K, Black RE; WHO Child Health Epidemiology Reference Group, 2005. WHO estimates of the causes of death in children. Lancet 365: 1147-1152.

2. Niehaus MD, Moore SR, Patrick PD, Derr LL, Lorntz B, Lima AA, Guerrant RL, 2002. Early childhood diarrhea is associated with diminished cognitive function 4 to 7 years later in children in a northeast Brazilian shantytown. Am J Trop Med Hyg 66: 590-593.

3. Berkman DS, Lescano AG, Gilman RH, Lopez SL, Black MM, 2002. Effects of stunting, diarrhoeal disease, and parasitic infection during infancy on cognition in late childhood: a follow-up study. Lancet 359: 564-571.

4. Lorntz B, Soares AM, Moore SR, Pinkerton R, Gansneder B, Bovbjerg VE, Guyatt H, Lima AM, Guerrant RL, 2006. Early childhood diarrhea predicts impaired school performance. Pediatr Infect Dis J 25: 513-520.
5. Steiner TS, Samie A, Guerrant RL, 2006. Infectious diarrhea: new pathogens and new challenges in developed and developing areas. Clin Infect Dis 43: 408-410.

6. Petri WA, Miller M, Binder HJ, Levine MM, Dillingham R, Guerrant RL,2008. Enteric infections, diarrhea, and their impact on function and development. J Clin Invest 118: 1277-1290.

7. Morris SS, Cousens SN, Kirkwood BR, Arthur P, Ross DA, 1996. Is prevalence of diarrhea a better predictor of subsequent mortality and weight gain than diarrhea incidence? Am J Epidemiol 144: 582-588.

8. Vila J, Vargas M, Casals C, Urassa H, Mshinda H, Schellemberg D, Gascon J, 1999. Antimicrobial resistance of diarrheagenic Escherichia coli isolated from children under the age of 5 years from Ifakara, Tanzania. Antimicrob Agents Chemother 43: 3022-3024.

9. Franzolin MR, Alves RC, Keller R, Gomes TA, Beutin L, Barreto ML, Milroy C, Strina A, Ribeiro H,Trabulsi LR, 2005. Prevalence of diarrheagenic Escherichia coli in children with diarrhea in Salvador, Bahia, Brazil. Mem Inst Oswaldo Cruz 100: 359-363.

10. Nguyen TV, Le PV, Le CH, Weintraub A, 2005. Antibiotic resistance in diarrheagenic Escherichia coli and Shigella strains isolated from children in Hanoi, Vietnam. Antimicrob Agents Chemother 49: 816-819.

11. Estrada-García T, Cerna JF, Pacheco-Gil L, Velázquez RF, Ochoa TJ, Torres J, DuPont HL, 2005. Drug-resistant diarrheogenic Escherichia coli, Mexico. Emerg Infect Dis 11: 1306-1308.

12. Nataro JP, Kaper JB, 1998. Diarrheagenic Escherichia coli. Clin Microbiol Rev 11: 142-201.

13. Guion CE, Ochoa TJ, Walker CM, Barletta F, Cleary TG, 2008. Diagnosis of the diarrheagenic Escherichia coli using melting curve analysis of a real-time multiplex polymerase chain reaction. J Clin Microbiol 46: 1752-1757.

14. Clinical Laboratory Standards Institute (CLSI), 2006. Performance Standards for Antimicrobial Susceptibility Testing. Sixteenth Informational Supplement. M100-S16. Vol. 26, No. 3. Wayne, PA: The Institute.

15. Pickering LK, 2004. Antimicrobial resistance among enteric pathogens. Semin Pediatr Infect Dis 15: 71-77.

16. Cabada MM, White AC Jr, 2008. Travelers' diarrhea: an update on susceptibility, prevention, and treatment. Curr Gastroenterol Rep 10: 473-479.

17. Leibovitz E, Janco J, Piglansky L, Press J, Yagupsky P, Reinhart H, Yaniv I, Dagan R, 2000. Oral ciprofloxacin vs. intramuscular ceftriaxone as empiric treatment of acute invasive diarrhea in children. Pediatr Infect Dis J 19: 1060-1067.

18. O'Ryan M, Prado V, Pickering LK, 2005. A millennium update on pediatric diarrheal illness in the developing world. Semin Pediatr Infect Dis 16: 125-136.

19. Vanden Eng J, Marcus R, Hadler JL, Imhoff B, Vugia DJ, Cieslak PR, Zell E, Deneen V, McCombs KG, Zansky SM, Hawkins MA, Besser RE, 2003. Consumer attitudes and use of antibiotics. Emerg Infect Dis 9: 1128-1135.

20. Magee JT, Pritchard EL, Fitzgerald KA, Dunstan FD, Howard AJ, 1999. Antibiotic prescribing and antibiotic resistance in community practice: retrospective study, 1996-8. BMJ 319: 1239-1240.

21. Bojalil R, Calva JJ,1994. Antibiotic misuse in diarrhea. A household survey in a Mexican community. J Clin Epidemiol 47: 147-156.

22. Bartoloni A, Cutts F, Leoni S, Austin CC, Mantella A, Guglielmetti P, Roselli M, Salazar E, Paradisi F, 1998. Patterns of antimicrobial use and antimicrobial resistance among healthy children in Bolivia. Trop Med Int Health 3: 116-123.

23. Howteerakul N, Higginbotham N, Dibley MJ, 2004. Antimicrobial use in children under five years with diarrhea in a central region province, Thailand. Southeast Asian J Trop Med Public Health 35: 181-187.

24. Binsztein N, Picandet AM, Notario R, Patrito E, De Lesa ME, De Petris A, Maurel D, Nader O, Rivas M, Szefner M, Vergara M, 1999. Antimicrobial resistance among species of Salmonella, Shigella, Escherichia, and Aeromonas isolated from children with diarrhea in 7 Argentinian centers [in Spanish]. Rev Latinoam Microbiol 41: 121-126.

25. Mandomando IM, Macete EV, Ruiz J, Sanz S, Abacassamo F, Vallès X, Sacarlal J, Navia MM, Vila J, Alonso PL, Gascon J, 2007. Etiology of diarrhea in children younger than 5 years of 
age admitted in a rural hospital of southern Mozambique. Am J Trop Med Hyg 76: 522-527.

26. Okeke IN, Laxminarayan R, Bhutta ZA, Duse AG, Jenkins P, O'Brien TF, Pablos-Mendez A, Klugman KP, 2005. Antimicrobial resistance in developing countries. Part I: recent trends and current status. Lancet Infect Dis 5: 481-493.

27. Okeke IN, Aboderin OA, Byarugaba DK, Ojo KK, Opintan JA, 2007. Growing problem of multidrug-resistant enteric pathogens in Africa. Emerg Infect Dis 13: 1640-1646.

28. Hien BT, Scheutz F, Cam PD, Serichantalergs O, Huong TT, Thu TM, Dalsgaard A, 2008. Diarrheagenic Escherichia coli and Shigella strains isolated from children in a hospital case-control study in Hanoi, Vietnam. J Clin Microbiol 46: 996-1004.

29. Lopes LM, Fabbricotti SH, Ferreira AJ, Kato MA, Michalski J, Scaletsky IC, 2005. Heterogeneity among strains of diffusely adherent Escherichia coli isolated in Brazil. J Clin Microbiol 43: 1968-1972.

30. Harrington SM, Dudley EG, Nataro JP, 2006. Pathogenesis of enteroaggregative Escherichia coli infection. FEMS Microbiol Lett 254: 12-18.
31. Brueggemann AB, 2006. Antibiotic resistance mechanisms among pediatric respiratory and enteric pathogens. Pediatr Infect Dis J 25: 969-973.

32. Barbosa TM, Levy SB, 2000. Differential expression of over 60 chromosomal genes in Escherichia coli by constitutive expression of MarA.J Bacteriol 182: 3467-3474.

33. Maira-Litrán T, Allison DG, Gilbert P, 2000. Expression of the multiple antibiotic resistance operon (mar) during growth of Escherichia coli as a biofilm. J Appl Microbiol 88: 243-247.

34. Gassama A, Aïdara-Kane A, Chainier D, Denis F, Ploy MC, 2004. Integron-associated antibiotic resistance in enteroaggregative and enteroinvasive Escherichia coli. Microb Drug Resist 10: 27-30.

35. Okeke IN, Steinrück H, Kanack KJ, Elliott SJ, Sundström L, Kaper JB, Lamikanra A, 2002. Antibiotic-resistant cell-detaching Escherichia coli strains from Nigerian children.J Clin Microbiol 40: 301-305.

36. Nwaneshiudu AI, Mucci T, Pickard DJ, Okeke IN, 2007. A second large plasmid encodes conjugative transfer and antimicrobial resistance in O119:H2 and some typical O111 enteropathogenic Escherichia coli strains. J Bacteriol 189: 6074-6079. 